\title{
Berry Curvature and Phonon Hall Effect
}

\author{
Tao Qin, ${ }^{1}$ Jianhui Zhou, ${ }^{1}$ and Junren $\mathrm{Shi}^{2}$ \\ ${ }^{1}$ Institute of Physics, Chinese Academy of Sciences, Beijing 100190, China \\ ${ }^{2}$ International Center for Quantum Materials, Peking University, Beijing 100871, China
}

\begin{abstract}
We show that an effective magnetic-field acting on phonons naturally emerges in phonon dynamics of magnetic solids, giving rise to the phonon Hall effect. A general formula of the intrinsic phonon Hall conductivity is derived by using the corrected Kubo formula with the energy magnetization contribution incorporated properly. We thus establish a direct connection between the phonon Hall effect and the intrinsic phonon band structure, i.e., phonon Berry curvature and phonon dispersions. Based on the formalism, we predict that phonon could also have quantum Hall effect in certain topological phonon systems. In the low temperature regime, we predict that the phonon Hall conductivity is proportional to $T^{3}$ for ordinary phonon systems, while that for the topological phonon system has the linear $T$ dependence with the quantized temperature coefficient.
\end{abstract}

PACS numbers: 66.70.-f, 72.20.Pa, 72.10.Bg, 72.15.Gd

\section{INTRODUCTION}

The phonon Hall effect (PHE) has been discovered recently in $\mathrm{Tb}_{3} \mathrm{Ga}_{5} \mathrm{O}_{12}(\mathrm{TGG})^{1}$.2. : a magnetized solid could give rise to a temperature difference between two edges of the sample in the direction transverse to both the magnetization and the driving thermal flow. The discovery of the Hall effect of the neutral carriers such as phonon has incited great theoretical interests $s^{3-7}$. Most of the theories relate the effect to the Raman spin-lattice coupling ${ }^{8}$, and the standard linear response theory, i.e., the Kubo formula ${ }^{3,5}-\underline{\underline{7}}$ or its equivalent $\underline{4}$, are employed to calculate the thermal Hall coefficient. These investigations, while all focusing on the intrinsic limit, have not yet revealed a simple connection between the phonon Hall effect and the intrinsic phonon band structure for a general system, as that done in electron systems $\stackrel{9}{ }$. There is also a natural and interesting question: could phonon systems have quantum (anomalous) Hall effect as well6,10,11?

In this paper, we establish a direct connection between the PHE and the intrinsic phonon band structure, i.e., phonon Berry curvature and phonon dispersions. To do this, first, we derive the general phonon dynamics applicable for magnetic solids, incorporating the MeadTruhlar term in the Born-Oppenheimer approximation ${ }^{12}$. The resulting dynamics contains an effective magnetic field acting on phonons, which gives rise to the PHE. It clarifies the microscopic origin of the spin-lattice coupling, and is readily amendable for the first principles calculation. Second, we calculate the thermal Hall coefficient of the system using the corrected Kubo formula, incorporating the contribution of the energy magnetization ${ }^{13,14}$, which is overlooked in all previous calculations. As the result, we obtain a general formula for calculating the intrinsic phonon Hall coefficient. Based on the formalism, we predict that phonon could also have quantum (anomalous) Hall effect in properly defined topological phonon systems. In the low temperature regime, we predict that the phonon Hall conductivity is proportional to $T^{3}$ for the ordinary phonon systems, while that for the topological phonon systems has the linear $T$ dependence with the quantized temperature coefficient.

This paper is organized as follow. In Sec. II, we discussed the general phonon dynamics in the magnetic field. In Sec. III, we presented our central formula for the phonon Hall conductivity. In Sec. IV, we gave the definition for the topological phonon systems and also discussed the possibility to realize it. In Sec. V, we show the $T^{3}$ law in the low temperature limit for the phonon Hall conductivity. In Sec. VI, we have a brief summary. We also include an Appendix section to show details of derivations.

\section{PHONON DYNAMICS OF MAGNETIC SOLIDS}

The Hamiltonian for the nucleus motion of a magnetic solid is determined by the complete form of the BornOppenheimer approximation ${ }^{12}$ :

$$
\hat{H}=\sum_{l \kappa} \frac{\left(-\mathrm{i} \hbar \nabla_{l \kappa}-\boldsymbol{A}_{l \kappa}(\{\boldsymbol{R}\})\right)^{2}}{2 M_{\kappa}}+V_{\mathrm{eff}}(\boldsymbol{R}),
$$

where $\nabla_{l \kappa}=\partial / \partial \boldsymbol{u}_{l \kappa}$, and $V_{\text {eff }}(\{\boldsymbol{R}\})=\tilde{E}_{\mathrm{e}}(\{\boldsymbol{R}\})+$ $E_{\mathrm{i}}(\{\boldsymbol{R}\})$ is the effective interacting potential for nuclei, including the direct Coulomb interaction between nuclei $E_{\mathrm{i}}(\{\boldsymbol{R}\})$, as well as the nucleus interaction mediated by electrons: $\tilde{E}_{\mathrm{e}}(\{\boldsymbol{R}\})=E_{0}(\{\boldsymbol{R}\})+$ $\left(\hbar^{2} / 2 M_{\kappa}\right) \sum_{l \kappa}\left[\left\langle\nabla_{l \kappa} \Phi_{0} \mid \nabla_{l \kappa} \Phi_{0}\right\rangle-\left|\left\langle\Phi_{0} \mid \nabla_{l \kappa} \Phi_{0}\right\rangle\right|^{2}\right]$, where $E_{0}(\{\boldsymbol{R}\})$ is the energy of the ground state $\left|\Phi_{0}(\{\boldsymbol{R}\})\right\rangle$ of the electron subsystem at the instantaneous nucleus positions $\{\boldsymbol{R}\}$. For the crystalline solid, the equilibrium positions of nuclei form a Bravais lattice, and we use $\{\boldsymbol{R}\} \equiv\left\{\boldsymbol{R}_{l \kappa}^{0}+\boldsymbol{u}_{l \kappa}, l=1 \ldots N ; \kappa=1 \ldots r\right\}$ with $\boldsymbol{R}_{l \kappa}^{0} \equiv \boldsymbol{R}_{l}^{0}+\boldsymbol{d}_{\kappa}$, where $\boldsymbol{R}_{l}^{0}$ is the center equilibrium position of $l$-th unit cell, $\boldsymbol{d}_{\kappa}$ is the equilibrium position of $\kappa$-th nucleus (with mass $M_{\kappa}$ ) related to the center, $\boldsymbol{u}_{l \kappa}$ is the corresponding vibration displacement, $N$ denotes 
the total number of the unit cells, and $r$ is the number of atoms in each unit cell.

The most notable feature of Eq. (1) is the presence of the vector potential $\boldsymbol{A}_{l \kappa}(\{\boldsymbol{R}\}) \equiv$ $\mathrm{i} \hbar\left\langle\Phi_{0}(\{\boldsymbol{R}\}) \mid \nabla_{l \kappa} \Phi_{0}(\{\boldsymbol{R}\})\right\rangle \quad\left(\boldsymbol{\nabla}_{l \kappa} \equiv \partial / \partial \boldsymbol{u}_{l \kappa}\right)$, as first pointed out by Mead and Truhlar ${ }^{12}$. In the modern language, the vector potential is related to the Berry phase ${ }^{15}$. The corresponding "physical field" at the limit $\boldsymbol{u}_{l \kappa} \rightarrow 0$ is:

$$
G_{\alpha \beta}^{\kappa \kappa^{\prime}}\left(\boldsymbol{R}_{l}^{0}-\boldsymbol{R}_{l^{\prime}}^{0}\right)=\left.2 \hbar \operatorname{Im}\left\langle\frac{\partial \Phi_{0}}{\partial u_{\beta, l^{\prime} \kappa^{\prime}}} \mid \frac{\partial \Phi_{0}}{\partial u_{\alpha, l \kappa}}\right\rangle\right|_{\boldsymbol{u}_{l \kappa} \rightarrow 0},
$$

where $\alpha, \beta=x, y, z$, and the translational symmetry dictates that it must be a function of $\boldsymbol{R}_{l}^{0}-\boldsymbol{R}_{l^{\prime}}^{0}$.

In the external magnetic field, the ion will experience two vector potentials 16 : one from the real magnetic field and the other from the Berry phase. The role of the latter is crucial here. In a single atom, there is exact cancellation between the two, or the neutral atom would be deflected in the magnetic field. Therefore, it is not right to deal with the ion in the magnetic field directly by the "minimal substitution"17. In the lattice system, the cancellation is not exact, and the spin-orbit coupling of the electron will give rise to nonzero $G_{\alpha \beta}^{\kappa \kappa^{\prime}}\left(\boldsymbol{R}_{l}^{0}-\boldsymbol{R}_{l^{\prime}}^{0}\right)$.

To proceed, we adopt the usual harmonic approximation by expanding $V_{\text {eff }}(\{\boldsymbol{R}\})$ to the second order of the vibration displacement $\boldsymbol{u}_{l \kappa} \underline{18}$. For the periodic lattice, it is more convenient to use the Fourier-transformed displacement $\boldsymbol{u}_{\kappa \boldsymbol{k}}=\sqrt{M_{\kappa} / N} \sum_{l} \boldsymbol{u}_{l \kappa} e^{-\mathrm{i} \boldsymbol{k} \cdot \boldsymbol{R}_{l \kappa}^{0}}$ with $\boldsymbol{k}$ being the quasi-momentum, and the Hamiltonian can be written as:

$$
\hat{H}=\frac{1}{2} \sum_{\boldsymbol{k}}\left(\hat{P}_{\boldsymbol{k}}^{\dagger} \hat{P}_{\boldsymbol{k}}+\hat{u}_{\boldsymbol{k}}^{\dagger} D_{\boldsymbol{k}} \hat{u}_{\boldsymbol{k}}\right)
$$

where $\hat{P}_{\boldsymbol{k}}$ and $\hat{u}_{\boldsymbol{k}}$ have $3 r$ components with $\hat{P}_{\kappa \alpha, \boldsymbol{k}} \equiv$ $-\mathrm{i} \hbar \partial / \partial u_{\kappa \alpha,-\boldsymbol{k}}-A_{\kappa \alpha, \boldsymbol{k}}$ and $\hat{u}_{\kappa \alpha, \boldsymbol{k}}, \alpha=x, y, z$ respectively, $A_{\kappa \alpha, \boldsymbol{k}}=\mathrm{i} \hbar\left\langle\Phi_{0} \mid \partial \Phi_{0} / \partial u_{\kappa \alpha,-\boldsymbol{k}}\right\rangle$, and $D_{\boldsymbol{k}}$ is the $3 r \times 3 r$ dynamical matrix ${ }^{18}$.

The momentum $\hat{P}_{\kappa \alpha, \boldsymbol{k}}$ has the commutation relation at the limit $\boldsymbol{u}_{l \kappa} \rightarrow 0$ :

$$
\begin{gathered}
{\left[\hat{P}_{\kappa \alpha, \boldsymbol{k}}, \hat{P}_{\kappa^{\prime} \beta, \boldsymbol{k}^{\prime}}^{\dagger}\right]=\mathrm{i} \hbar G_{\kappa \alpha, \kappa^{\prime} \beta}(\boldsymbol{k}) \delta_{\boldsymbol{k} \boldsymbol{k}^{\prime}}} \\
G_{\kappa \alpha, \kappa^{\prime} \beta}(\boldsymbol{k})=\frac{1}{\sqrt{M_{\kappa} M_{\kappa^{\prime}}}} \sum_{l} G_{\alpha \beta}^{\kappa \kappa^{\prime}}\left(R_{l}^{0}\right) e^{-\mathrm{i} \boldsymbol{k} \cdot\left(\boldsymbol{R}_{l}^{0}+\boldsymbol{d}_{\kappa \kappa^{\prime}}\right)},
\end{gathered}
$$

where $\boldsymbol{d}_{\kappa \kappa^{\prime}} \equiv \boldsymbol{d}_{\kappa}-\boldsymbol{d}_{\kappa^{\prime}} . G_{\kappa \alpha, \kappa^{\prime} \beta}(\boldsymbol{k})$ acts like an effective magnetic field for the phonon dynamics. Using Eq. (2) and (5), the quantity is readily calculable for the real materials using the first principles approach.

The general phonon dynamics for a magnetic solid can now be determined. From Eq. (3), the linearized canonical equations of motion read:

$$
\begin{aligned}
& \dot{\hat{u}}_{\boldsymbol{k}}=\hat{P}_{\boldsymbol{k}}, \\
& \dot{\hat{P}}_{\boldsymbol{k}}=-D_{\boldsymbol{k}} \hat{u}_{\boldsymbol{k}}+G_{\boldsymbol{k}} \hat{P}_{\boldsymbol{k}},
\end{aligned}
$$

where $G_{\boldsymbol{k}}$ is a $3 r \times 3 r$ matrix with the component $G_{\kappa \alpha, \nu \beta}(\boldsymbol{k})$.

The corresponding eigen-equation is:

$$
\omega_{\boldsymbol{k} i} \psi_{\boldsymbol{k} i}=\left[\begin{array}{cc}
0 & \mathrm{i} \\
-\mathrm{i} D_{\boldsymbol{k}} & \mathrm{i} G_{\boldsymbol{k}}
\end{array}\right] \psi_{\boldsymbol{k} i} \equiv \tilde{H}_{\boldsymbol{k}} \psi_{\boldsymbol{k} i}
$$

where $\psi_{\boldsymbol{k} i}$ is the $i$ th eigen solution, and $\omega_{\boldsymbol{k} i}$ is the corresponding eigen frequency. For the non-hermitian $\tilde{H}_{\boldsymbol{k}}$, we define $\bar{\psi}_{\boldsymbol{k} i}=\psi_{\boldsymbol{k} i}^{\dagger} \tilde{D}_{\boldsymbol{k}}$ with $\tilde{D}_{\boldsymbol{k}} \equiv\left[\begin{array}{cc}D_{\boldsymbol{k}} & 0 \\ 0 & I_{3 r \times 3 r}\end{array}\right]$ and $I_{3 r \times 3 r}$ is unit matrix of dimension $3 r \times 3 r$, and $\psi_{\boldsymbol{k} i}$ is normalized by $\bar{\psi}_{\boldsymbol{k} i} \psi_{\boldsymbol{k} j}=\delta_{i j}$. Note that the $6 r$ eigensolutions can be divided into two groups of $3 r$ positive and negative energy branches, and the two are related by $\omega_{\boldsymbol{k} i}^{(-)}=-\omega_{-\boldsymbol{k} i}^{(+)}$and $\psi_{\boldsymbol{k} i}^{(-)}=\psi_{-\boldsymbol{k} i}^{(+) *}$ with $1 \leq i \leq 3 r$. This is a result of the symmetries $G_{\boldsymbol{k}}^{*}=G_{-\boldsymbol{k}}$ and $D_{\boldsymbol{k}}^{*}=D_{-\boldsymbol{k}}$. In the following we would only use the positive energy branches and drop the superscript $(+)$ for brevity.

The Hamiltonian Eq. (3) can be diagonalized with the basis $\psi_{\boldsymbol{k}}$. By defining the field operator $\hat{\Psi}_{\boldsymbol{k}} \equiv\left(\hat{u}_{\boldsymbol{k}}, \hat{P}_{\boldsymbol{k}}\right)^{T}$, Eq. (3) can be rewritten as $\hat{H}=(1 / 2) \sum_{\boldsymbol{k}} \hat{\bar{\Psi}}_{\boldsymbol{k}} \hat{\Psi}_{\boldsymbol{k}}$ with $\hat{\bar{\Psi}}_{\boldsymbol{k}} \equiv \hat{\Psi}_{\boldsymbol{k}}^{\dagger} \tilde{D}_{\boldsymbol{k}}$. Introducing the transformation

$$
\hat{\Psi}_{\boldsymbol{k}}=\sum_{i=1}^{3 r} \sqrt{\hbar \omega_{\boldsymbol{k} i}} \psi_{\boldsymbol{k} i} \hat{a}_{\boldsymbol{k} i}+\sqrt{\hbar \omega_{-\boldsymbol{k} i}} \psi_{-\boldsymbol{k} i}^{*} \hat{a}_{-\boldsymbol{k} i}^{\dagger}
$$

with $\left[\hat{a}_{\boldsymbol{k} i}, \hat{a}_{\boldsymbol{k} j}^{\dagger}\right]=\delta_{i j}, i, j=1 \ldots 3 r$, we can recover all the commutation relations, and diagonalize the Hamiltonian to:

$$
\hat{H}=\sum_{\boldsymbol{k} ; i=1}^{3 r} \hbar \omega_{\boldsymbol{k} i}\left(\hat{a}_{\boldsymbol{k} i}^{\dagger} \hat{a}_{\boldsymbol{k} i}+\frac{1}{2}\right)
$$

Similar to the electronic dynamics in magnetic solids ${ }^{19}$, the intrinsic phonon band structure is determined not only by the phonon dispersions, but also by the Berry connections of the phonon bands. We can define the phonon Berry connection as $\boldsymbol{A}_{\boldsymbol{k} i} \equiv \mathrm{i} \bar{\psi}_{\boldsymbol{k} i}\left(\partial \psi_{\boldsymbol{k} i} / \partial \boldsymbol{k}\right)$, and the corresponding Berry curvature as,

$$
\boldsymbol{\Omega}_{\boldsymbol{k} i}=-\operatorname{Im}\left[\frac{\partial \bar{\psi}_{\boldsymbol{k} i}}{\partial \boldsymbol{k}} \times \frac{\partial \psi_{\boldsymbol{k} i}}{\partial \boldsymbol{k}}\right]
$$

We will show that the phonon Berry curvatures $\boldsymbol{\Omega}_{\boldsymbol{k} i}$ and the phonon dispersions $\omega_{\boldsymbol{k} i}$ will fully determine the intrinsic phonon Hall conductivity. On the other hand, the inter-band Berry curvatures proposed in some of previous studies are not needed in general $\underline{6}$.

\section{PHONON HALL CONDUCTIVITY}

Following the established general procedure ${ }^{13}$, we can calculate the thermal Hall coefficient contributed by phonons. For a magnetic system, the transport thermal 
Hall coefficient includes two parts of contributions: the usual linear response contribution $\kappa^{\text {Kubo }}$ and the contribution from the energy magnetization $M_{E}$ :

$$
\kappa_{x y}^{\mathrm{tr}}=\kappa_{x y}^{\mathrm{Kubo}}+\frac{2 M_{E}^{z}}{T V},
$$

where $V$ is the total volume of the system, and $T$ is the temperature. $\boldsymbol{M}_{E}$ is the circulation of the phonon energy current, and the reason of the circulation can only be attributed to our effective magnetic field.

\section{A. Kubo contribution $\kappa_{x y}^{K u b o}$}

$\kappa_{x y}^{\mathrm{Kubo}}$ is determined by the usual Kubo formula ${ }^{20}$ :

$\kappa_{x y}^{\mathrm{Kubo}}=\frac{1}{V k_{B} T^{2}} \lim _{s \rightarrow 0} \lim _{\boldsymbol{q} \rightarrow 0} \int_{0}^{\infty} \mathrm{d} t e^{-s t}\left\langle\hat{J}_{E,-\boldsymbol{q}}^{y} ; \hat{J}_{E, \boldsymbol{q}}^{x}(t)\right\rangle$,

where $\hat{\boldsymbol{J}}_{E, \boldsymbol{q}}$ is the Fourier transformed energy current operator in the wave-vector $\boldsymbol{q}$, and $\langle;\rangle$ denotes the Kubo canonical correlation ${ }^{21}$.

Using the procedure developed by $\mathrm{Hardy}^{22}$, and with the harmonic approximation and in the small $\boldsymbol{q}$ limit, we find that, [See Appendix A1]:

$$
\hat{\boldsymbol{J}}_{E, \boldsymbol{q}}=\frac{1}{8} \sum_{\boldsymbol{k}} \hat{\bar{\Psi}}_{\boldsymbol{k}}\left(\tilde{\boldsymbol{V}}_{\boldsymbol{k}}+\tilde{\boldsymbol{V}}_{\boldsymbol{k}+\boldsymbol{q}}\right) \hat{\Psi}_{\boldsymbol{k}+\boldsymbol{q}}+(\text { h.c. }, \boldsymbol{q} \rightarrow-\boldsymbol{q})
$$

where $\tilde{\boldsymbol{V}}_{\boldsymbol{k}} \equiv \boldsymbol{\nabla}_{\boldsymbol{k}} \tilde{H}_{\boldsymbol{k}}$, and (h.c., $\boldsymbol{q} \rightarrow-\boldsymbol{q}$ ) denotes the hermitian conjugation of the first term after replacing $\boldsymbol{q}$ by $-\boldsymbol{q}$.

With the phonon current operator $\hat{\boldsymbol{J}}_{E, \boldsymbol{q}}$, we have [See Appendix B],

$$
\kappa_{x y}^{\mathrm{Kubo}}=\frac{\hbar}{V T} \sum_{\boldsymbol{k} ; i=1}^{3 r} \mathcal{M}_{\boldsymbol{k} i}^{z} \omega_{\boldsymbol{k} i}\left(n_{\boldsymbol{k} i}+\frac{1}{2}\right),
$$

where $\boldsymbol{M}_{\boldsymbol{k} i}=\operatorname{Im}\left[\frac{\partial \bar{\psi}_{\boldsymbol{k} i}}{\partial \boldsymbol{k}} \times \tilde{H}_{\boldsymbol{k}} \frac{\partial \psi_{\boldsymbol{k} i}}{\partial \boldsymbol{k}}\right]$, and $n_{\boldsymbol{k} i} \equiv n_{B}\left(\hbar \omega_{\boldsymbol{k} i}\right)$ is the Bose-Einstein distribution. The presence of the "zero point" contribution (the extra $\frac{1}{2}$ inside the parenthesis) is due to the phonon number non-conserving terms (e.g., $a_{\boldsymbol{k} i} a_{\boldsymbol{k}+\boldsymbol{q} j}$ or $a_{\boldsymbol{k} i}^{\dagger} a_{\boldsymbol{k}+\boldsymbol{q} j}^{\dagger}$ ) in the energy current operator, which were often improperly dropped in many previous calculations ${ }^{3-5}$. As a result of the zero point contribution, $\kappa_{x y}^{\text {Kubo }}$ diverges when $T \rightarrow 0$.

\section{B. Phonon energy magnetization $M_{E}^{z}$}

The unphysical divergence can be removed by the second term of Eq. (12). The energy magnetization is determined as follows 13 :

$$
\begin{aligned}
& 2 M_{E}^{z}-T \frac{\partial M_{E}^{z}}{\partial T}=\tilde{M}_{E}^{z}, \\
& \tilde{M}_{E}^{z}=\left.\frac{1}{k_{B} T} \mathrm{i} \frac{\partial}{\partial q_{y}}\left\langle\hat{h}_{-\boldsymbol{q}} ; \hat{J}_{E, \boldsymbol{q}}^{x}\right\rangle\right|_{\boldsymbol{q} \rightarrow 0},
\end{aligned}
$$

where $h_{\boldsymbol{q}}$ is the Fourier transformed energy density operator:

$$
\hat{h}_{\boldsymbol{q}}=\frac{1}{2} \sum_{\boldsymbol{k}} \hat{\bar{\Psi}}_{\boldsymbol{k}} \hat{\Psi}_{\boldsymbol{k}+\boldsymbol{q}}
$$

The energy current operator defined in Eq. (14) should satisfy the scaling law necessary for the applicability ${ }^{13}$ of Eqs. (16 17) [See Appendix A2].

For the phonon energy magnetization in Eqs. (16 17), we have [See Appendix C]:

$$
\begin{aligned}
\tilde{M}_{E}^{z}=-\frac{\hbar}{2} \sum_{\boldsymbol{k} ; i=1}^{3 r} \omega_{\boldsymbol{k} i} & {\left[\Omega_{\boldsymbol{k} i}^{z} \omega_{\boldsymbol{k} i}^{2} n_{\boldsymbol{k} i}^{\prime}\right.} \\
& \left.+\mathcal{M}_{\boldsymbol{k} i}^{z}\left(2 n_{\boldsymbol{k} i}+\omega_{\boldsymbol{k} i} n_{\boldsymbol{k} i}^{\prime}+1\right)\right]
\end{aligned}
$$

where $n_{\boldsymbol{k} i}^{\prime}=\partial n_{\boldsymbol{k} i} / \partial \omega_{\boldsymbol{k} i} . M_{E}^{z}$ is obtained by integrating over the temperature $T$ with the boundary condition that $2 M_{E}^{z}$ coincides with $\tilde{M}_{E}^{z}$ when $T=0$.

\section{Phonon Hall conductivity}

We are on a position to present our central result for the phonon Hall conductivity.

$$
\kappa_{x y}^{\mathrm{tr}}=-\frac{\left(\pi k_{B}\right)^{2}}{3 h} Z_{\mathrm{ph}} T-\frac{1}{T} \int d \epsilon \epsilon^{2} \sigma_{x y}(\epsilon) \frac{d n(\epsilon)}{d \epsilon},
$$

where

$$
\sigma_{x y}(\epsilon)=-\frac{1}{V \hbar} \sum_{\hbar \omega_{\boldsymbol{k} i} \leq \epsilon} \Omega_{\boldsymbol{k} i}^{z}
$$

and

$$
Z_{\mathrm{ph}}=\frac{2 \pi}{V} \sum_{\boldsymbol{k} ; i=1}^{3 r} \Omega_{\boldsymbol{k} i}^{z}
$$

Equation (20) gives the general formula of the intrinsic phonon Hall conductivity for a magnetic solid. As expected, the intrinsic phonon Hall conductivity is fully determined by the dispersions and the Berry curvatures.

In the following, we show how the topological term emerges naturally in Eq. (20).

First, we express $\kappa_{x y}^{\text {Kubo }}, \tilde{M}_{E}^{z \text {,inter }}$ and $\tilde{M}_{E}^{z \text {,intra }}$ as:

$$
\begin{aligned}
\kappa_{x y}^{\text {Kubo }} & =\frac{1}{2 T} \int d \epsilon \epsilon m_{1 z}^{6 r}(\epsilon) n(\epsilon) \\
\tilde{M}_{E}^{z, \text { inter }} & =-\frac{1}{2} \int d \epsilon \epsilon m_{1 z}^{6 r}(\epsilon) n(\epsilon) \\
\tilde{M}_{E}^{z, \text { intra }} & =-\frac{1}{4} \int d \epsilon\left(m_{1 z}^{6 r}(\epsilon)-\frac{1}{\hbar} \epsilon \tilde{\sigma}_{x y}^{6 r}(\omega)\right) \epsilon^{2} \frac{\partial n(\epsilon)}{\partial \epsilon}
\end{aligned}
$$


where

$$
\begin{aligned}
& m_{1 z}^{6 r}(\epsilon)=\frac{1}{V} \sum_{\boldsymbol{k}, i=1}^{6 r} \mathcal{M}_{\boldsymbol{k} i}^{z} \delta\left(\epsilon-\hbar \omega_{\boldsymbol{k} i}\right) \\
& \tilde{\sigma}_{x y}^{6 r}(\epsilon)=-\frac{1}{V \hbar} \sum_{\boldsymbol{k}, i=1}^{6 r} \Omega_{\boldsymbol{k} i}^{z} \delta\left(\epsilon-\hbar \omega_{\boldsymbol{k} i}\right) .
\end{aligned}
$$

So the real energy magnetization $M_{E}^{z}$ is:

$$
\begin{aligned}
M_{E}^{z}=- & \frac{V}{4} \int d \epsilon \epsilon m_{1 z}^{6 r}(\epsilon) \frac{1}{\beta^{2}} \int^{\beta} d \beta^{\prime} \beta^{\prime}\left(2 n(\epsilon)+\epsilon \frac{\partial n(\epsilon)}{\partial \epsilon}\right) \\
& +\frac{V}{4} \int d \epsilon \epsilon^{3} \tilde{\sigma}_{x y}^{6 r}(\epsilon) \frac{1}{\beta^{2}} \int^{\beta} d \beta^{\prime} \beta^{\prime} \frac{\partial n(\epsilon)}{\partial \epsilon},
\end{aligned}
$$

where the boundary condition is that $2 M_{E}^{z}$ coincides with $\tilde{M}_{E}^{z}$ when $T=0$. There is no indefinity in Eq. (28), for the thermodynamic quantity ${ }^{13} \boldsymbol{M}_{s}=\boldsymbol{M}_{E} / T$ should be zero in the high temperature limit. We can change the integration over $\beta^{\prime}$ into the integration over $\epsilon$ and we have:

$$
\begin{aligned}
\kappa_{x y}^{\mathrm{tr}} & =\kappa_{x y}^{\mathrm{Kubo}}+\frac{2 M_{E}^{z}}{T V} \\
& =\frac{1}{2 T} \int d \epsilon \tilde{\sigma}_{x y}^{6 r}(\epsilon)\left(-2 \int^{\epsilon} d x x n(x)+\epsilon^{2} n(\epsilon)\right) .
\end{aligned}
$$

We can define $\tilde{\sigma}_{x y}^{6 r}(\epsilon)=\frac{d \sigma_{x y}^{6 r}(\epsilon)}{d \epsilon}$, so we have:

$$
\begin{array}{r}
\kappa_{x y}^{\mathrm{tr}}=\left.\frac{1}{2 T} \sigma_{x y}^{6 r}(\epsilon)\left(-2 \int^{\epsilon} d x x n(x)+\epsilon^{2} n(\epsilon)\right)\right|_{-\infty} ^{\infty} \\
-\frac{1}{2 T} \int d \epsilon \sigma_{x y}^{6 r}(\epsilon) \epsilon^{2} \frac{d n(\epsilon)}{d \epsilon}
\end{array}
$$

The first term in Eq. (31) is zero, for the following reasons:

$$
\begin{aligned}
\sigma_{x y}^{6 r}(\epsilon) & =\int_{-\infty}^{\epsilon} d x \tilde{\sigma}_{x y}^{6 r}(x), \\
\sigma_{x y}^{6 r}(\infty) & =0, \\
\sigma_{x y}^{6 r}(-\infty) & =0 .
\end{aligned}
$$

We can show Eq. (33) by the properties of the phonon Berry curvature: $\Omega_{\boldsymbol{k} i}^{(-)}=-\Omega_{-\boldsymbol{k} i}^{(+)}$where $1 \leq i \leq 3 r$, because of the symmetry properties of $\psi_{\boldsymbol{k} i}$. Eq. (34) is zero because of the integration limit. Therefore,

$$
\kappa_{x y}^{\mathrm{tr}}=-\frac{1}{2 T} \int d \epsilon \epsilon^{2} \sigma_{x y}^{6 r}(\epsilon) \frac{d n(\epsilon)}{d \epsilon} .
$$

Next, we express $\kappa_{x y}^{\mathrm{tr}}$ with the $3 r$ positive energy bands.

$$
\begin{aligned}
\sigma_{x y}^{6 r}(\epsilon) & =\int_{-\infty}^{0} d x \tilde{\sigma}_{x y}^{6 r}(x)+\int_{0}^{\epsilon} d x \tilde{\sigma}_{x y}^{6 r}(x), \\
& =-\frac{Z_{\mathrm{ph}}}{2 \pi \hbar}+\int_{0}^{\epsilon} d x \tilde{\sigma}_{x y}^{6 r}(x) .
\end{aligned}
$$

Using our definition in Eq. (21), we can write Eq. (37) as:

$$
\sigma_{x y}^{6 r}(\epsilon)= \begin{cases}-\frac{Z_{\mathrm{ph}}}{2 \pi \hbar}+\sigma_{x y}(\epsilon), & \epsilon>0 \\ -\frac{Z_{\mathrm{ph}}}{2 \pi \hbar}+\sigma_{x y}(-\epsilon) . & \epsilon<0\end{cases}
$$

Therefore,

$$
\begin{aligned}
\kappa_{x y}^{\mathrm{tr}}= & -\frac{1}{2 T} \int_{0}^{\infty} d \epsilon \epsilon^{2}\left(-\frac{Z_{\mathrm{ph}}}{2 \pi \hbar}+\sigma_{x y}(\epsilon)\right) \frac{d n(\epsilon)}{d \epsilon} \\
& -\frac{1}{2 T} \int_{-\infty}^{0} d \epsilon \epsilon^{2}\left(-\frac{Z_{\mathrm{ph}}}{2 \pi \hbar}+\sigma_{x y}(-\epsilon)\right) \frac{d n(\epsilon)}{d \epsilon} .
\end{aligned}
$$

Finally, after some simple algebra we obtain the phonon Hall conductivity in Eq. (20) and the topological term emerges naturally.

\section{TOPOLOGICAL PHONON SYSTEM}

The first term in Eq. (20) is of the topological nature, determined by the global phonon band structures. This is different from the second term which is only determined by the low energy sectors of the phonon bands limited by $k_{B} T$. For the two-dimensional system (2D), $Z_{\text {ph }}$ is the Chern number, quantized as an integer. For the three-dimensional systems $(3 \mathrm{D}), Z_{\mathrm{ph}}$ is quantized 23 in the unit $G_{z} / 2 \pi$, where $G_{z}$ is the $z$-component of a reciprocal lattice lattice vector $\boldsymbol{G}$. As the result, the phonon Hall conductivity has a topological contribution with the quantized linear temperature coefficient in the unit $\left(\pi k_{B}\right)^{2} / 3 h(2 \mathrm{D})$ or $\pi k_{B}^{2} G_{z} / 6 \pi h(3 \mathrm{D})$.

Not surprisingly, most of the phonon systems have $Z_{\mathrm{ph}}=0$. It is natural to define the topological phonon systems as those with:

$$
Z_{\mathrm{ph}} \neq 0
$$

It puts a stringent constraint on what the real topological phonon system is: it requires that the sum of the Chern numbers of all phonon bands must be nonzero. The previous theoretical studies have successes in constructing the phonon systems with non-zero Chern numbers of individual bands $6,10,11$. However, these models rely on the reorganization of the phonon bands within the positive energy branches, so still have zero $Z_{\mathrm{ph}}$. They are not topological phonon systems in the stringent sense, nor will they manifest unusual phonon Hall conductivity. To realize the real topological phonon system, we need to look for insulating materials with spin-orbit coupling so strong that the resulting effective magnetic field can inter-mix and re-organize the phonon bands between the positive and negative energy branches. A realistic lattice model for realizing the topological phonon system is an interesting topic for future investigations.

We note that the value of the topological contribution to the thermal Hall conductivity is actually the 
same as the longitudinal thermal conductivity of a dielectric quantum wire with $Z_{\mathrm{ph}}$ acoustic phonon modes ${ }^{24-26}$. This is because the topological phonon systems have chiral edge phonon modes that behave just like the $1 \mathrm{D}$ acoustic phonon modes. The edge-bulk correspondence picture in the topological phonon system is very similar as that in quantum Hall systems. On the other hand, unlike the phonons in a quantum wire, phonons in the chiral edge modes can not be backscattered without coupling to the bulk. This will make topological phonon Hall effect more robust against imperfections than the quantized thermal conduction in $1 \mathrm{D}$ quantum wire. The latter had been observed experimentally ${ }^{25}$.

\section{LOW TEMPERATURE LIMIT}

In the low temperature, only the low energy phonon modes are relevant for the ordinary systems. It is thus sufficient to consider the long wave acoustic phonon modes.

\section{A. Constraint for the effective magnetic field}

An important constraint for this case is:

$$
\sum_{l \kappa \kappa^{\prime}} G_{\alpha \beta}^{\kappa \kappa^{\prime}}\left(\boldsymbol{R}_{l}^{0}\right)=0
$$

This can be verified directly by using the effective magnetic filed in Eq. (2). As we will show, this is still true even when we explicitly consider the external magnetic field because of the overall charge neutrality. It is easy to see that:

$$
\begin{aligned}
& \sum_{l \kappa \kappa^{\prime}} G_{\alpha \beta}^{\kappa \kappa^{\prime}}\left(\boldsymbol{R}_{l}^{0}\right) \\
= & \left.\frac{2 \hbar}{N} \operatorname{Im}\left\langle\frac{\partial \Phi_{0}\left(\left\{\boldsymbol{R}^{0}+\boldsymbol{u}_{0}\right\}\right)}{\partial u_{0 \beta}} \mid \frac{\partial \Phi_{0}\left(\left\{\boldsymbol{R}^{0}+\boldsymbol{u}_{0}\right\}\right)}{\partial u_{0 \alpha}}\right\rangle\right|_{\boldsymbol{u}_{0} \rightarrow 0} \\
& +Z_{\text {tot }} e \epsilon_{\alpha \beta \gamma} B_{\gamma},
\end{aligned}
$$

where $\Phi_{0}\left(\left\{\boldsymbol{R}^{0}+\boldsymbol{u}_{0}\right\}\right)$ is the ground state wave-function of the electron subsystem when the whole system is displaced with $\boldsymbol{u}_{0}$, and $Z_{\text {tot }}$ is the total charge number of the nuclei in an unit cell. We have assumed that there is an external magnetic field $\boldsymbol{B}$. Since the displacement is equivalent to a re-definition of the origin, we have:

$$
\begin{gathered}
\Phi_{0}\left(\left\{\boldsymbol{R}^{0}+\boldsymbol{u}_{0}\right\},\{\boldsymbol{r}\}\right)=\exp \left[-\mathrm{i} \frac{e}{2 \hbar}\left(\boldsymbol{B} \times \boldsymbol{u}_{0}\right) \cdot\left(\sum_{i} \boldsymbol{r}_{i}\right)\right] \\
\cdot \Phi_{0}\left(\left\{\boldsymbol{R}^{0}\right\},\left\{\boldsymbol{r}-\boldsymbol{u}_{0}\right\}\right),
\end{gathered}
$$

where $\{\boldsymbol{r}\} \equiv\left\{\boldsymbol{r}_{1}, \boldsymbol{r}_{2} \ldots \boldsymbol{r}_{N_{e}}\right\}$ denotes the coordinates of the electron. We have:

$$
\begin{aligned}
& \frac{\partial \Phi_{0}\left(\left\{\boldsymbol{R}^{0}+\boldsymbol{u}_{0}\right\},\{\boldsymbol{r}\}\right)}{\partial \boldsymbol{u}_{0}} \\
= & -\frac{\mathrm{i}}{\hbar} \exp \left[-\mathrm{i} \frac{e}{2 \hbar}\left(\boldsymbol{B} \times \boldsymbol{u}_{0}\right) \cdot\left(\sum_{i} \boldsymbol{r}_{i}\right)\right] \\
& \cdot \sum_{i}\left(\hat{\boldsymbol{p}}_{i}-\frac{e}{2} \boldsymbol{B} \times \boldsymbol{r}_{i}\right) \Phi_{0}\left(\left\{\boldsymbol{R}^{0}\right\},\left\{\boldsymbol{r}-\boldsymbol{u}_{0}\right\}\right) .
\end{aligned}
$$

Substituting Eq. (45) into (43), one can easily verify that the first term and the second term of the right hand side of Eq. (43) exactly cancel each other due to the overall charge neutrality.

\section{B. $T^{3}$ law}

It is easy to see that the phonon dynamics with the constraint always has three acoustic modes that have zero energy at $\boldsymbol{k}=0$, in consistent with the general requirement of global translational symmetry. This remedies an important issue of the widely adopted phenomenological model of Raman spin-lattice coupling, which has nonvanishing coupling constant even in the long wave limit, inducing nonzero acoustic phonon energies at $\boldsymbol{k}=0$. We will see that the constraint will change the theoretical expectation of the low temperature behavior of the phonon Hall conductivity.

With this constraint in mind, we can write down the general Hamiltonian for the long-wave acoustic phonons of an isotropic continuous medium 27 :

$$
\hat{H}=\int \mathrm{d} \boldsymbol{x}\left[\frac{\hat{\boldsymbol{P}}(\boldsymbol{x})^{2}}{2 \rho}+\frac{\mu_{1}}{2} \boldsymbol{\nabla} \hat{\boldsymbol{u}} \cdot \boldsymbol{\nabla} \hat{\boldsymbol{u}}+\frac{\mu_{2}}{2}(\boldsymbol{\nabla} \cdot \hat{\boldsymbol{u}})^{2}\right],
$$

where $\hat{\boldsymbol{P}}(\boldsymbol{x})=-\mathrm{i} \hbar \delta / \delta \hat{\boldsymbol{u}}(\boldsymbol{x})-\boldsymbol{A}[\hat{\boldsymbol{u}}], \boldsymbol{u}(\boldsymbol{x})$ is the vibration displacement, $\mu_{1}$ and $\mu_{2}$ are elastic constants, $\rho$ is the mass density. The symmetry dictates: $\boldsymbol{A}[\hat{\boldsymbol{u}}]=\gamma_{1} \boldsymbol{\nabla} \boldsymbol{\nabla} \cdot(\boldsymbol{M} \times \hat{\boldsymbol{u}})+\gamma_{2} \boldsymbol{\nabla}^{2}(\boldsymbol{M} \times \hat{\boldsymbol{u}})$, where $\boldsymbol{M}$ is the magnetization of the system, and $\gamma_{1}$ and $\gamma_{2}$ are coupling constants characterizing the spin-lattice coupling. With the Fourier transformation, we can identify $D_{\alpha \beta}(\boldsymbol{k})=(1 / \rho)\left(\mu_{1} \delta_{\alpha \beta} k^{2}+\mu_{2} k_{\alpha} k_{\beta}\right)$ and $G_{\alpha \beta}(\boldsymbol{k})=$ $(1 / \rho) \sum_{\gamma} \epsilon_{\alpha \beta \gamma}\left[-\gamma_{1} k_{\gamma} \boldsymbol{k} \cdot \boldsymbol{M}+\left(\gamma_{1}+2 \gamma_{2}\right) \boldsymbol{k}^{2} M_{\gamma}\right]$ with $k=|\boldsymbol{k}|$ and $\alpha, \beta, \gamma=x, y, z$. We can then adopt our general formula for calculating the phonon Hall conductivity.

To the first order of the magnetization $\boldsymbol{M}$, we obtain the phonon dispersions: $\omega_{\boldsymbol{k} 1}=c_{L} k$ and $\omega_{\boldsymbol{k} 2(3)}=c_{T} k \pm$ $\gamma_{2} k \boldsymbol{k} \cdot \boldsymbol{M}$, where $c_{T}=\sqrt{\mu_{1} / \rho}$ and $c_{L}=\sqrt{\left(\mu_{1}+2 \mu_{2}\right) / \rho}$ are transverse and longitudinal phonon velocities, respec- 
tively. The phonon Berry curvatures are:

$$
\begin{aligned}
\boldsymbol{\Omega}_{\boldsymbol{k} 1} & =-\frac{g_{1}\left(k^{2} \boldsymbol{M}+\boldsymbol{k} \boldsymbol{k} \cdot \boldsymbol{M}\right)}{k^{3}}, \\
\boldsymbol{\Omega}_{\boldsymbol{k} 2(3)} & = \pm \frac{\boldsymbol{k}}{k^{3}}+\frac{g_{2}\left(k^{2} \boldsymbol{M}+\boldsymbol{k} \boldsymbol{k} \cdot \boldsymbol{M}\right)}{k^{3}}
\end{aligned}
$$

where $g_{1}=\left(\gamma_{1}+2 \gamma_{2}\right)\left(1+3 \delta^{2}\right) /\left(2 c_{T} \delta\left(\delta^{2}-1\right)\right), g_{2}=$ $g_{1} \delta\left(3+\delta^{2}\right) /\left(2\left(1+3 \delta^{2}\right)\right)$, and $\delta=c_{L} / c_{T}$.

Using Eq. (20), we determine the phonon Hall conductivity:

$\kappa_{x y}^{\mathrm{tr}}=\frac{4 \pi^{2} k_{B}^{4}}{45 c_{T}^{3} \hbar^{3}}\left[1-\frac{\gamma_{1}+2 \gamma_{2}}{2 \gamma_{2}} \frac{4 \delta^{3}+\delta^{2}+\delta+1}{\delta^{3}\left(\delta^{2}+\delta+1\right)}\right] \gamma_{2} M_{z} T^{3}$,

where we assume $M$ is along the $z$-direction and the Debye energy $\hbar \omega_{D} \gg k_{B} T$. We can see that at the low temperature, $\kappa_{x y}^{\mathrm{tr}}$ is proportional to $T^{3}$, instead of $T$ as proposed in the previous studies ${ }^{3}$.

We can obtain insights from the above calculation on how the phonon would be deflected by the effective magnetic field. We can see from Eqs. (47) and (48) that, on different branches, the phonon will experience different "reciprocal space magnetic fields". The corresponding anomalous velocity of the phonon is proportional to $\boldsymbol{\Omega}_{\boldsymbol{k} i} \times \boldsymbol{\nabla} T$, similar to that for electrons $\frac{19}{}$. The net deflection direction will be perpendicular to both the directions of the magnetization and the temperature gradient.

A few comments concerning the disorder effect 28 : In analogue to the anomalous Hall effect of electron systems ${ }^{29}$, we generally expect the total phonon Hall coefficient can be decomposed to $\kappa_{x y}^{\mathrm{tr}}=\kappa_{x y}^{\mathrm{in}}+\kappa_{x y}^{\mathrm{sj}}+\kappa_{x y}^{\text {skew }}$, where $\kappa_{x y}^{\text {in }}$ is the intrinsic phonon Hall conductivity we calculate in Eq. (20), and the disorder will introduce the side jump contribution $\kappa_{x y}^{\mathrm{sj}}$ and the skew scattering contribution $\kappa_{x y}^{\text {skew }}$. However, there is an important difference between the phonon system and electron system: the mean free path of phonon in TGG had been determined to be $\sim 1 \mathrm{~mm}^{30}$, much longer than its electron counterpart. Moreover, in the low temperature limit, the dominant contribution to the thermal conductivity is from the long wave phonons which don't "see" the disorder ${ }^{31}$. We thus expect that these disorder correction is less important in phonon systems, and the $T^{3}$ law of the phonon Hall conductivity will survive.

\section{SUMMARY}

In summary, we establish the general phonon dynamics for the magnetic solids. Based on the dynamics, we propose a general theory of the PHE. Using the corrected Kubo formula, we link the intrinsic phonon Hall conductivity to the phonon Berry curvature. The general formula suggests that phonon could also have quantum Hall effect, and our theory presents a rigorous definition of the topological phonon system. We predict that the phonon
Hall conductivity of the ordinary phonon system is proportional to $T^{3}$ at the low temperature, while that for the topological phonon system has the linear $T$ dependence with the quantized temperature coefficient.

\section{APPENDIX}

\section{A. Phonon energy current operator and its scaling} law

\section{Phonon energy current operator}

We follow Hardy ${ }^{22}$ to derive the phonon energy current operator. The Hamiltonian density is:

$$
\hat{h}(\boldsymbol{x})=\frac{1}{2} \sum_{l \kappa}\left\{\Delta\left(\boldsymbol{x}-\boldsymbol{R}_{l \kappa}\right) \hat{H}_{l \kappa}+\text { h.c. }\right\},
$$

where $\hat{H}_{l \kappa}=\frac{\hat{\boldsymbol{P}}_{l \kappa}^{2}}{2 M_{\kappa}}+\hat{V}_{l \kappa}$ and $\hat{\boldsymbol{P}}_{l \kappa}=-\mathrm{i} \hbar \nabla_{l \kappa}-\boldsymbol{A}_{l \kappa}$, and $\Delta(\boldsymbol{x})$ is a localized function near $\boldsymbol{x}=0$ with $\int \mathrm{d} \boldsymbol{x} \Delta(\boldsymbol{x})=1$. We adopt the Harmonic approximation for $\hat{V}_{l \kappa}: \hat{V}_{l \kappa}=\frac{1}{2} \sum_{l^{\prime} \kappa^{\prime} \alpha \beta} \hat{u}_{l \kappa \alpha} D_{\alpha \beta}^{\kappa \kappa^{\prime}}\left(\boldsymbol{R}_{l}^{0}-\boldsymbol{R}_{l^{\prime}}^{0}\right) \hat{u}_{l^{\prime} \kappa^{\prime} \beta}$ where $D_{\alpha \beta}^{\kappa \kappa^{\prime}}\left(\boldsymbol{R}_{l}^{0}-\boldsymbol{R}_{l^{\prime}}^{0}\right)$ is the dynamical matrix. The Hamiltonian is $\hat{H}=\int \mathrm{d} \boldsymbol{x} \hat{h}(\boldsymbol{x})=\sum_{l \kappa} H_{l \kappa}$. The energy current operator is defined by the energy conservation equation:

$$
\dot{\hat{h}}(\boldsymbol{x})+\boldsymbol{\nabla} \cdot \hat{\boldsymbol{J}}_{E}(\boldsymbol{x})=0,
$$

where $\hat{\boldsymbol{J}}_{E}(\boldsymbol{x})$ is the phonon energy current operator, and $\dot{\hat{h}}(\boldsymbol{x}) \equiv(1 / \mathrm{i} \hbar)[\hat{h}(\boldsymbol{x}), \hat{H}]$.

We can express $[\hat{h}(\boldsymbol{x}), \hat{H}]$ as a divergence. We have:

$$
\begin{aligned}
\frac{\mathrm{i}}{\hbar}[\hat{h}(\boldsymbol{x}), \hat{H}] & =\frac{\mathrm{i}}{2 \hbar} \sum_{l \kappa, l^{\prime} \kappa^{\prime}} \Delta\left(\boldsymbol{x}-\boldsymbol{R}_{l \kappa}\right)\left[\hat{H}_{l \kappa}, \hat{H}_{l^{\prime} \kappa^{\prime}}\right] \\
& + \text { h.c. }+\cdots,
\end{aligned}
$$

where "..." denotes the higher order terms such as $\left[\Delta\left(\boldsymbol{x}-\boldsymbol{R}_{l \kappa}\right), \frac{\hat{\boldsymbol{P}}_{l^{\prime}{ }^{\prime}}^{2}}{2 M_{\kappa^{\prime}}}\right] \hat{H}_{l \kappa}$, which will be a cubic product of $\hat{\boldsymbol{u}}_{l \kappa}$ and $\hat{\boldsymbol{P}}_{l^{\prime} \kappa^{\prime}}$. Noting that,

$$
\begin{gathered}
{\left[\hat{H}_{l \kappa}, \hat{H}_{l^{\prime} \kappa^{\prime}}\right]=\left[\frac{\hat{\boldsymbol{P}}_{l \kappa}^{2}}{2 M_{\kappa}}, \hat{V}_{l^{\prime} \kappa^{\prime}}\right]+\left[\hat{V}_{l \kappa}, \frac{\hat{\boldsymbol{P}}_{l^{\prime} \kappa^{\prime}}^{2}}{2 M_{\kappa^{\prime}}}\right]} \\
+\frac{\mathrm{i} \hbar}{2 M_{\kappa} M_{\kappa^{\prime}}} \sum_{\alpha \beta} G_{\alpha \beta}^{\kappa \kappa^{\prime}}\left(\boldsymbol{R}_{l}^{0}-\boldsymbol{R}_{l^{\prime}}^{0}\right)\left(\hat{P}_{l \kappa}^{\alpha} \hat{P}_{l^{\prime} \kappa^{\prime}}^{\beta}+\hat{P}_{l^{\prime} \kappa^{\prime}}^{\beta} \hat{P}_{l \kappa}^{\alpha}\right),
\end{gathered}
$$

and interchanging subscripts $l \kappa$ and $l^{\prime} \kappa^{\prime}$, we have: 


$$
\begin{aligned}
& \frac{\mathrm{i}}{\hbar}[\hat{h}(\boldsymbol{x}), \hat{H}] \\
= & \frac{\mathrm{i}}{2 \hbar} \sum_{l \kappa, l^{\prime} \kappa^{\prime}}\left[\Delta\left(\boldsymbol{x}-\boldsymbol{R}_{l \kappa}\right)-\Delta\left(\boldsymbol{x}-\boldsymbol{R}_{l^{\prime} \kappa^{\prime}}\right)\right]\left(\left[\frac{\hat{\boldsymbol{P}}_{l \kappa}^{2}}{2 M_{\kappa}}, \hat{V}_{l^{\prime} \kappa^{\prime}}\right]\right. \\
& \left.+\frac{\mathrm{i} \hbar}{2 M_{\kappa} M_{\kappa^{\prime}}} \sum_{\alpha \beta} G_{\alpha \beta}^{\kappa \kappa^{\prime}}\left(\boldsymbol{R}_{l}^{0}-\boldsymbol{R}_{l^{\prime}}^{0}\right) \hat{P}_{l \kappa}^{\alpha} \hat{P}_{l^{\prime} \kappa^{\prime}}^{\beta}\right)+ \text { h.c.. }
\end{aligned}
$$

Inserting the expansion

$$
\begin{array}{r}
\Delta\left(\boldsymbol{x}-\boldsymbol{R}_{l \kappa}\right)-\Delta\left(\boldsymbol{x}-\boldsymbol{R}_{l^{\prime} \kappa^{\prime}}\right) \approx \frac{1}{2}\left(\boldsymbol{R}_{l^{\prime} \kappa^{\prime}}^{0}-\boldsymbol{R}_{l \kappa}^{0}\right) \\
\cdot\left(\frac{\partial \Delta\left(\boldsymbol{x}-\boldsymbol{R}_{l^{\prime} \kappa^{\prime}}^{0}\right)}{\partial \boldsymbol{x}}+\frac{\partial \Delta\left(\boldsymbol{x}-\boldsymbol{R}_{l \kappa}^{0}\right)}{\partial \boldsymbol{x}}\right),
\end{array}
$$

into Eq. (A5), we obtain:

$$
\frac{\mathrm{i}}{\hbar}[\hat{h}(\boldsymbol{x}), \hat{H}]=\boldsymbol{\nabla} \cdot \hat{\boldsymbol{J}}_{E}(\boldsymbol{x}),
$$

where

$$
\begin{gathered}
\hat{\boldsymbol{J}}_{E}(\boldsymbol{x})=\frac{\mathrm{i}}{4 \hbar} \sum_{l \kappa, l^{\prime} \kappa^{\prime}}\left(\boldsymbol{R}_{l^{\prime} \kappa^{\prime}}^{0}-\boldsymbol{R}_{l \kappa}^{0}\right)\left(\Delta\left(\boldsymbol{x}-\boldsymbol{R}_{l^{\prime} \kappa^{\prime}}^{0}\right)\right. \\
\left.+\Delta\left(\boldsymbol{x}-\boldsymbol{R}_{l \kappa}^{0}\right)\right)\left(\left[\frac{\hat{\boldsymbol{P}}_{l \kappa}^{2}}{2 M_{\kappa}}, \hat{V}_{l^{\prime} \kappa^{\prime}}\right]+\right. \\
\left.+\frac{\mathrm{i} \hbar}{2 M_{\kappa} M_{\kappa^{\prime}}} \sum_{\alpha \beta} G_{\alpha \beta}^{\kappa \kappa^{\prime}}\left(\boldsymbol{R}_{l}^{0}-\boldsymbol{R}_{l^{\prime}}^{0}\right) \hat{P}_{l \kappa}^{\alpha} \hat{P}_{l^{\prime} \kappa^{\prime}}^{\beta}\right)+ \text { h.c. }
\end{gathered}
$$

Doing the Fourier transformation $\hat{\boldsymbol{J}}_{E, \boldsymbol{q}}=$ $\int d \boldsymbol{x} \hat{\boldsymbol{J}}_{E}(\boldsymbol{x}) e^{-\mathrm{i} \boldsymbol{q} \cdot \boldsymbol{x}}$, we have:

$$
\begin{array}{r}
\hat{\boldsymbol{J}}_{E, \boldsymbol{q}}=-\frac{\mathrm{i}}{8} \Delta_{\boldsymbol{q}} \sum_{\boldsymbol{k}}\left(\hat{\boldsymbol{P}}_{\boldsymbol{k}}^{\dagger} \nabla_{\boldsymbol{k}}\left(D_{\boldsymbol{k}}+D_{\boldsymbol{k}+\boldsymbol{q}}\right) \hat{\boldsymbol{u}}_{\boldsymbol{k}+\boldsymbol{q}}\right. \\
\left.-\hat{\boldsymbol{P}}_{\boldsymbol{k}}^{\dagger} \nabla_{\boldsymbol{k}}\left(G_{\boldsymbol{k}}+G_{\boldsymbol{k}+\boldsymbol{q}}\right) \hat{\boldsymbol{P}}_{\boldsymbol{k}+\boldsymbol{q}}\right),
\end{array}
$$

where $D_{\boldsymbol{k}}$ is the dynamic matrix with components $D_{\kappa \alpha, \kappa^{\prime} \beta}(\boldsymbol{k})=\frac{1}{\sqrt{M_{\kappa} M_{\kappa^{\prime}}}} \sum_{l} D_{\alpha \beta}^{\kappa \kappa^{\prime}}\left(R_{l}^{0}\right) e^{-\mathrm{i} \boldsymbol{k} \cdot\left(\boldsymbol{R}_{l}^{0}+\boldsymbol{d}_{\kappa \kappa^{\prime}}\right)} . \quad$ In the small $\boldsymbol{q}$ limit, $\Delta_{\boldsymbol{q}} \rightarrow 1$. We obtain:

Using the identities: $\boldsymbol{\nabla}_{\boldsymbol{k}} \tilde{H}_{\boldsymbol{k}}=\tilde{D}_{\boldsymbol{k}} \boldsymbol{\nabla}_{\boldsymbol{k}} \tilde{H}_{\boldsymbol{k}}$ and $\boldsymbol{\nabla}_{\boldsymbol{k}} \tilde{H}_{\boldsymbol{k}}^{\dagger}=$ $\left(\nabla_{\boldsymbol{k}} \tilde{H}_{\boldsymbol{k}}^{\dagger}\right) \tilde{D}_{\boldsymbol{k}}$, we obtain Eq. (14).

$$
\begin{aligned}
\hat{\boldsymbol{J}}_{E, \boldsymbol{q}}=\frac{1}{8} \sum_{\boldsymbol{k}} \hat{\Psi}_{\boldsymbol{k}}^{\dagger} & \left(\boldsymbol{\nabla}_{\boldsymbol{k}}\left(\tilde{H}_{\boldsymbol{k}}+\tilde{H}_{\boldsymbol{k}+\boldsymbol{q}}\right)\right. \\
& \left.+\nabla_{\boldsymbol{k}}\left(\tilde{H}_{\boldsymbol{k}}^{\dagger}+\tilde{H}_{\boldsymbol{k}+\boldsymbol{q}}^{\dagger}\right)\right) \hat{\Psi}_{\boldsymbol{k}+\boldsymbol{q}} .
\end{aligned}
$$

\section{Scaling law for the energy current operator}

In order to calculate the energy magnetization, we need to verify that the energy current satisfies the scaling law 13 :

$$
\hat{\boldsymbol{J}}_{E}^{\psi}(\boldsymbol{x})=(1+\psi(\boldsymbol{x}))^{2} \hat{\boldsymbol{J}}_{E}(\boldsymbol{x})+\mathcal{O}\left[\boldsymbol{\nabla}^{(2)} \psi(\boldsymbol{x})\right]
$$

in the presence of the gravitational field $\psi(\boldsymbol{r})$ that modifies the local Hamiltonian density by:

$$
\hat{h}^{\psi}(\boldsymbol{x})=\frac{1}{2} \sum_{l \kappa}\left\{(1+\psi(\boldsymbol{x})) \Delta\left(\boldsymbol{x}-\boldsymbol{R}_{l \kappa}\right) \hat{H}_{l \kappa}+H . c .\right\} .
$$

Similar to the derivation in Sec. VIIA1, we have:

$$
\boldsymbol{\nabla} \cdot \hat{\boldsymbol{J}}_{E}^{\psi}(\boldsymbol{x})=\frac{\mathrm{i}}{\hbar}\left[\hat{h}^{\psi}(\boldsymbol{x}), \hat{H}^{\psi}\right]
$$


and:

$$
\begin{aligned}
\frac{\mathrm{i}}{\hbar}\left[\hat{h}^{\psi}(\boldsymbol{x}), \hat{H}^{\psi}\right] & =\frac{\mathrm{i}}{4} \int d \boldsymbol{x}^{\prime}(1+\psi(\boldsymbol{x}))\left(1+\psi\left(\boldsymbol{x}^{\prime}\right)\right) \Gamma\left(\boldsymbol{x}, \boldsymbol{x}^{\prime}\right) \\
& \approx \frac{\mathrm{i}}{4} \int d \boldsymbol{x}^{\prime}\left[(1+\psi(\boldsymbol{x}))^{2} \Gamma\left(\boldsymbol{x}, \boldsymbol{x}^{\prime}\right)+(1+\psi(\boldsymbol{x}))\left(\boldsymbol{x}^{\prime}-\boldsymbol{x}\right) \cdot \frac{\partial \psi(\boldsymbol{x})}{\partial \boldsymbol{x}} \Gamma\left(\boldsymbol{x}, \boldsymbol{x}^{\prime}\right)\right. \\
& \left.+\frac{1}{2}(1+\psi(\boldsymbol{x})) \sum_{\mu \nu}\left(x_{\mu}^{\prime}-x_{\mu}\right)\left(x_{\nu}^{\prime}-x_{\nu}\right) \frac{\partial^{2} \psi(\boldsymbol{x})}{\partial x_{\mu} \partial x_{\nu}} \Gamma\left(\boldsymbol{x}, \boldsymbol{x}^{\prime}\right)+\mathcal{O}\left[\boldsymbol{\nabla}^{(3)} \psi(\boldsymbol{x})\right]\right] .
\end{aligned}
$$

with

$$
\Gamma\left(\boldsymbol{x}, \boldsymbol{x}^{\prime}\right)=\frac{1}{\hbar} \sum_{l \kappa, l^{\prime} \kappa^{\prime}} \Delta\left(\boldsymbol{x}-\boldsymbol{R}_{l \kappa}\right)\left\{\Delta\left(\boldsymbol{x}^{\prime}-\boldsymbol{R}_{l^{\prime} \kappa^{\prime}}\right),\left[\hat{H}_{l \kappa}, \hat{H}_{l^{\prime} \kappa^{\prime}}\right]\right\}+\text { h.c. }+\cdots
$$

In Eq. (A17), we also ignore the terms which will lead to the cubic products of $\hat{\boldsymbol{u}}_{l \kappa}$ and $\hat{\boldsymbol{P}}_{l^{\prime} \kappa^{\prime}}$ in the energy current operator. For the first term in Eq. (A15), after the integration over $\boldsymbol{x}^{\prime}$ and repeating the derivation from Eq. (A3) to Eq. (A7), we can show:

$$
\frac{\mathrm{i}}{4} \int d \boldsymbol{x}^{\prime}(1+\psi(\boldsymbol{x}))^{2} \Gamma\left(\boldsymbol{x}, \boldsymbol{x}^{\prime}\right)=(1+\psi(\boldsymbol{x}))^{2} \boldsymbol{\nabla} \cdot \hat{\boldsymbol{J}}_{E}(\boldsymbol{x}) .
$$

For the second term in Eq. (A15):

$$
\begin{aligned}
& \frac{\mathrm{i}}{4} \int d \boldsymbol{x}^{\prime}(1+\psi(\boldsymbol{x}))\left(\boldsymbol{x}^{\prime}-\boldsymbol{x}\right) \cdot \frac{\partial \psi(\boldsymbol{x})}{\partial \boldsymbol{x}} \Gamma\left(\boldsymbol{x}, \boldsymbol{x}^{\prime}\right) \\
= & \frac{\mathrm{i}}{4 \hbar}(1+\psi(\boldsymbol{x})) \frac{\partial \psi(\boldsymbol{x})}{\partial \boldsymbol{x}} \cdot \sum_{l \kappa, l^{\prime} \kappa^{\prime}} \Delta\left(\boldsymbol{x}-\boldsymbol{R}_{l \kappa}\right)\left\{\boldsymbol{R}_{l^{\prime} \kappa^{\prime}}^{0}-\boldsymbol{R}_{l \kappa}^{0},\left[\hat{H}_{l \kappa}, \hat{H}_{l^{\prime} \kappa^{\prime}}\right]\right\}, \\
= & 2(1+\psi(\boldsymbol{x})) \frac{\partial \psi(\boldsymbol{x})}{\partial \boldsymbol{x}} \cdot \hat{\boldsymbol{J}}_{E}(\boldsymbol{x}) .
\end{aligned}
$$

For the third term in Eq. (A16), we have:

$$
\begin{aligned}
& \frac{\mathrm{i}}{4} \int d \boldsymbol{x}^{\prime}(1+\psi(\boldsymbol{x})) \sum_{\mu \nu}\left(x_{\mu}^{\prime}-x_{\mu}\right)\left(x_{\nu}^{\prime}-x_{\nu}\right) \frac{\partial^{2} \psi(\boldsymbol{x})}{\partial x_{\mu} \partial x_{\nu}} \Gamma\left(\boldsymbol{x}, \boldsymbol{x}^{\prime}\right) \\
= & \frac{\mathrm{i}}{2 \hbar}(1+\psi(\boldsymbol{x})) \sum_{l \kappa, l^{\prime} \kappa^{\prime}, \mu \nu} \frac{\partial^{2} \psi(\boldsymbol{x})}{\partial x_{\mu} \partial x_{\nu}} \Delta\left(\boldsymbol{x}-\boldsymbol{R}_{l \kappa}\right)\left(R_{l^{\prime} \kappa^{\prime} \mu}^{0}-R_{l \kappa \mu}^{0}\right)\left(R_{l^{\prime} \kappa^{\prime} \nu}^{0}-R_{l \kappa \nu}^{0}\right)\left[\hat{H}_{l \kappa}, \hat{H}_{l^{\prime} \kappa^{\prime}}\right]+\text { h.c. }, \\
= & \frac{\mathrm{i}}{4 \hbar}(1+\psi(\boldsymbol{x})) \sum_{l \kappa, l^{\prime} \kappa^{\prime}, \mu \nu} \frac{\partial^{2} \psi(\boldsymbol{x})}{\partial x_{\mu} \partial x_{\nu}}\left[\Delta\left(\boldsymbol{x}-\boldsymbol{R}_{l \kappa}\right)-\Delta\left(\boldsymbol{x}-\boldsymbol{R}_{l^{\prime} \kappa^{\prime}}\right)\right]\left(R_{l^{\prime} \kappa^{\prime} \mu}^{0}-R_{l \kappa \mu}^{0}\right)\left(R_{l^{\prime} \kappa^{\prime} \nu}^{0}-R_{l \kappa \nu}^{0}\right)\left[\hat{H}_{l \kappa}, \hat{H}_{l^{\prime} \kappa^{\prime}}\right]+\text { h.c. } .
\end{aligned}
$$

Noting the expansion in Eq. (A6), Eq. (A24) doesn't have a contribution to the current at the long wave limit.

Combining Eqs. (A18 A21), we obtain Eq. (A11).

\section{B. Derivation details for $\kappa_{x y}^{\text {Kubo }}$ in Eq. (15)}

Direct calculation of the Kubo formula leads to:

$\kappa_{x y}^{\text {Kubo }}=\frac{\hbar}{32 V T} \sum_{\boldsymbol{k} ; i, j=1}^{6 r} \frac{\operatorname{Im}\left(\mathcal{V}_{\boldsymbol{k} i j}^{x} \mathcal{V}_{\boldsymbol{k} j i}^{y}-\mathcal{V}_{\boldsymbol{k} i j}^{y} \mathcal{V}_{\boldsymbol{k} j i}^{x}\right) \omega_{\boldsymbol{k} i} \omega_{\boldsymbol{k} j} n_{\boldsymbol{k} i}}{\left(\omega_{\boldsymbol{k} i}-\omega_{\boldsymbol{k} j}\right)^{2}}$, where $\mathcal{V}_{\boldsymbol{k} i j} \equiv 2\left(\bar{\psi}_{\boldsymbol{k} i} \frac{\partial \tilde{H}_{\boldsymbol{k}}}{\partial \boldsymbol{k}} \psi_{\boldsymbol{k} j}+\psi_{\boldsymbol{k} i}^{\dagger} \frac{\partial \tilde{H}_{\boldsymbol{k}}^{\dagger}}{\partial \boldsymbol{k}} \bar{\psi}_{\boldsymbol{k} j}^{\dagger}\right)$.

It is easy to verify:

$$
\bar{\psi}_{\boldsymbol{k} i} \frac{\partial \tilde{H}_{\boldsymbol{k}}}{\partial k_{x}} \psi_{\boldsymbol{k} j}=\left(\omega_{\boldsymbol{k} j}-\omega_{\boldsymbol{k} i}\right) \bar{\psi}_{\boldsymbol{k} i} \frac{\partial \psi_{\boldsymbol{k} j}}{\partial k_{x}}+\frac{\partial \omega_{\boldsymbol{k} i}}{\partial k_{x}} \delta_{i j},
$$

so:

$\mathcal{V}_{\boldsymbol{k} i j}^{x}=4 \frac{\partial \omega_{\boldsymbol{k} i}}{\partial k_{x}} \delta_{i j}+2\left(\omega_{\boldsymbol{k} j}-\omega_{\boldsymbol{k} i}\right)\left(\bar{\psi}_{\boldsymbol{k} i} \frac{\partial \psi_{\boldsymbol{k} j}}{\partial k_{x}}+\psi_{\boldsymbol{k} i}^{\dagger} \frac{\partial \bar{\psi}_{\boldsymbol{k} j}^{\dagger}}{\partial k_{x}}\right)$.

Note here $i \neq j$, so we have: 


\section{Derivation details for $\tilde{M}_{E}^{z}$ in Eq. (19)}

$$
\begin{aligned}
& \sum_{j=1}^{6 r} \frac{\mathcal{V}_{\boldsymbol{k} i j}^{x} \mathcal{V}_{\boldsymbol{k} j i}^{y} \omega_{\boldsymbol{k} i} \omega_{\boldsymbol{k} j}}{\left(\omega_{\boldsymbol{k} i}-\omega_{\boldsymbol{k} j}\right)^{2}}= 4 \omega_{\boldsymbol{k} i}\left(\frac{\partial \bar{\psi}_{\boldsymbol{k} i}}{\partial k_{x}} \tilde{H}_{\boldsymbol{k}} \frac{\partial \psi_{\boldsymbol{k} i}}{\partial k_{y}}\right. \\
&+\frac{\partial \psi_{\boldsymbol{k} i}^{\dagger}}{\partial k_{x}} \tilde{H}_{\boldsymbol{k}}^{\dagger} \frac{\partial \bar{\psi}_{\boldsymbol{k} i}^{\dagger}}{\partial k_{y}}+\frac{\partial \psi_{\boldsymbol{k} i}^{\dagger}}{\partial k_{x}} \tilde{H}_{\boldsymbol{k}}^{\dagger} \tilde{D}_{\boldsymbol{k}} \frac{\partial \psi_{\boldsymbol{k} i}}{\partial k_{y}} \\
&\left.+\frac{\partial \bar{\psi}_{\boldsymbol{k} i}}{\partial k_{x}} \tilde{H}_{\boldsymbol{k}} \tilde{D}_{\boldsymbol{k}}^{-1} \frac{\partial \bar{\psi}_{\boldsymbol{k} i}^{\dagger}}{\partial k_{y}}\right)
\end{aligned}
$$

where we have used $\bar{\psi}_{\boldsymbol{k} i} \frac{\partial \psi_{\boldsymbol{k} j}}{\partial k_{x}}=-\frac{\partial \bar{\psi}_{\boldsymbol{k} i}}{\partial k_{x}} \psi_{\boldsymbol{k} j}, \psi_{\boldsymbol{k} j}^{\dagger} \frac{\partial \bar{\psi}_{\boldsymbol{k} i}^{\dagger}}{\partial k_{x}}=$ $-\frac{\partial \psi_{\boldsymbol{k}_{j}}^{\dagger}}{\partial k_{x}} \bar{\psi}_{\boldsymbol{k} i}^{\dagger}$, and $\tilde{H}_{\boldsymbol{k}}=\sum_{j} \omega_{\boldsymbol{k} j} \psi_{\boldsymbol{k} j} \bar{\psi}_{\boldsymbol{k} j}$ and its hermitian conjugate. We can further simplify the last two terms in Eq. (A28) using $\tilde{D}_{\boldsymbol{k}} \frac{\partial \psi_{\boldsymbol{k} i}}{\partial k_{y}}=\frac{\partial \bar{\psi}_{\boldsymbol{k} i}^{\dagger}}{\partial k_{y}}-\frac{\partial \tilde{D}_{\boldsymbol{k}}}{\partial k_{y}} \psi_{\boldsymbol{k} i}$ and $\tilde{D}_{\boldsymbol{k}}^{-1} \frac{\partial \bar{\psi}_{\boldsymbol{k} i}^{\dagger}}{\partial k_{y}}=\frac{\partial \psi_{\boldsymbol{k} i}}{\partial k_{y}}-\frac{\partial \tilde{D}_{\boldsymbol{k}}^{-1}}{\partial k_{y}} \bar{\psi}_{\boldsymbol{k} i}^{\dagger}$, and we obtain:

$$
\begin{aligned}
& \frac{\partial \psi_{\boldsymbol{k} i}^{\dagger}}{\partial k_{x}} \tilde{H}_{\boldsymbol{k}}^{\dagger} \tilde{D}_{\boldsymbol{k}} \frac{\partial \psi_{\boldsymbol{k} i}}{\partial k_{y}}+\frac{\partial \bar{\psi}_{\boldsymbol{k} i}}{\partial k_{x}} \tilde{H}_{\boldsymbol{k}} \tilde{D}_{\boldsymbol{k}}^{-1} \frac{\partial \bar{\psi}_{\boldsymbol{k} i}^{\dagger}}{\partial k_{y}} \\
= & \frac{\partial \psi_{\boldsymbol{k} i}^{\dagger}}{\partial k_{x}} \tilde{H}_{\boldsymbol{k}}^{\dagger} \frac{\partial \bar{\psi}_{\boldsymbol{k} i}^{\dagger}}{\partial k_{y}}+\frac{\partial \bar{\psi}_{\boldsymbol{k} i}}{\partial k_{x}} \tilde{H}_{\boldsymbol{k}} \frac{\partial \psi_{\boldsymbol{k} i}}{\partial k_{y}} \\
- & \frac{\partial \psi_{\boldsymbol{k} i}^{\dagger}}{\partial k_{x}} \tilde{H}_{\boldsymbol{k}}^{\dagger} \frac{\partial \tilde{D}_{\boldsymbol{k}}}{\partial k_{y}} \psi_{\boldsymbol{k} i}-\frac{\partial \bar{\psi}_{\boldsymbol{k} i}}{\partial k_{x}} \tilde{H}_{\boldsymbol{k}} \frac{\partial \tilde{D}_{\boldsymbol{k}}^{-1}}{\partial k_{y}} \bar{\psi}_{\boldsymbol{k} i}^{\dagger}
\end{aligned}
$$

The second term in Eq. (A30): $\frac{\partial \bar{\psi}_{\boldsymbol{k} i}}{\partial k_{x}} \tilde{H}_{\boldsymbol{k}} \frac{\partial \tilde{D}_{\boldsymbol{k}}^{-1}}{\partial k_{y}} \bar{\psi}_{\boldsymbol{k} i}^{\dagger}=$ $\psi_{\boldsymbol{k} i}^{\dagger} \frac{\partial D_{\boldsymbol{k}}}{\partial k_{x}} \tilde{H}_{\boldsymbol{k}} \frac{\partial \tilde{D}_{\boldsymbol{k}}^{-1}}{\partial k_{y}} \bar{\psi}_{\boldsymbol{k} i}^{\dagger}-\frac{\partial \psi_{\boldsymbol{k} i}^{\dagger}}{\partial k_{x}} \tilde{D}_{\boldsymbol{k}} \tilde{H}_{\boldsymbol{k}} \tilde{D}_{\boldsymbol{k}}^{-1} \frac{\partial \tilde{D}_{\boldsymbol{k}}}{\partial k_{y}} \psi_{\boldsymbol{k} i}=$ $-\frac{\partial \psi_{\boldsymbol{k} i}^{\dagger}}{\partial k_{x}} \tilde{H}_{\boldsymbol{k}}^{\dagger} \frac{\partial \tilde{D}_{\boldsymbol{k}}}{\partial k_{y}} \psi_{\boldsymbol{k} i}, \quad$ and $\quad \frac{\partial \tilde{D}_{\boldsymbol{k}}}{\partial k_{x}} \tilde{H}_{\boldsymbol{k}} \frac{\partial \tilde{D}_{\boldsymbol{k}}^{-1}}{\partial k_{y}}=0$ for $\frac{\partial \tilde{D}_{\boldsymbol{k}}}{\partial k_{x}}=\left[\begin{array}{cc}\frac{\partial D_{k}}{\partial k_{x}} & 0 \\ 0 & 0\end{array}\right]$, and then the two terms in Eq. (A30) vanish. Substituting Eq. (A29) into Eq. (A28), we have:

$$
\begin{aligned}
& \sum_{j=1}^{6 r} \frac{\operatorname{Im}\left(\mathcal{V}_{\boldsymbol{k} i j}^{x} \mathcal{V}_{\boldsymbol{k} j i}^{y}\right) \omega_{\boldsymbol{k} i} \omega_{\boldsymbol{k} j}}{\left(\omega_{\boldsymbol{k} i}-\omega_{\boldsymbol{k} j}\right)^{2}} \\
= & 8 \omega_{\boldsymbol{k} i} \operatorname{Im}\left(\frac{\partial \bar{\psi}_{\boldsymbol{k} i}}{\partial k_{x}} \tilde{H}_{\boldsymbol{k}} \frac{\partial \psi_{\boldsymbol{k} i}}{\partial k_{y}}-\frac{\partial \bar{\psi}_{\boldsymbol{k} i}}{\partial k_{y}} \tilde{H}_{\boldsymbol{k}} \frac{\partial \psi_{\boldsymbol{k} i}}{\partial k_{x}}\right) .
\end{aligned}
$$

Finally, we obtain:

$$
\begin{aligned}
\kappa_{x y}^{\mathrm{Kubo}=}= & \frac{\hbar}{2 V T} \operatorname{Im} \sum_{\boldsymbol{k}, i=1}^{6 r}\left(\frac{\partial \bar{\psi}_{\boldsymbol{k} i}}{\partial k_{x}} \tilde{H}_{\boldsymbol{k}} \frac{\partial \psi_{\boldsymbol{k} i}}{\partial k_{y}}-\frac{\partial \bar{\psi}_{\boldsymbol{k} i}}{\partial k_{y}} \tilde{H}_{\boldsymbol{k}} \frac{\partial \psi_{\boldsymbol{k} i}}{\partial k_{x}}\right) \\
& \cdot \omega_{\boldsymbol{k} i} n_{\boldsymbol{k} i}, \\
= & \frac{\hbar}{2 V T} \operatorname{Im} \sum_{\boldsymbol{k}, i=1}^{3 r}\left(\frac{\partial \bar{\psi}_{\boldsymbol{k} i}}{\partial k_{x}} \tilde{H}_{\boldsymbol{k}} \frac{\partial \psi_{\boldsymbol{k} i}}{\partial k_{y}}-\frac{\partial \bar{\psi}_{\boldsymbol{k} i}}{\partial k_{y}} \tilde{H}_{\boldsymbol{k}} \frac{\partial \psi_{\boldsymbol{k} i}}{\partial k_{x}}\right) \\
& \cdot \omega_{\boldsymbol{k} i}\left(2 n_{\boldsymbol{k} i}+1\right) .
\end{aligned}
$$

From Eq. (A32) to Eq. A33, we have used the symmetry properties of $\omega_{\boldsymbol{k} i}$ and $\psi_{\boldsymbol{k} i}$, so we come to Eq. (15).
After a direct calculation of the canonical correlation function in Eq. (17), we obtain:

$$
\begin{array}{r}
\tilde{M}_{E}^{z}=\frac{\mathrm{i} \hbar}{16} \frac{\partial}{\partial q_{y}} \sum_{\boldsymbol{k} ; i, j=1}^{6 r} S_{\boldsymbol{k}+\boldsymbol{q}, \boldsymbol{k} j i}\left(\mathcal{V}_{\boldsymbol{k}, \boldsymbol{k}+\boldsymbol{q} i j}^{x}+\mathcal{V}_{\boldsymbol{k}+\boldsymbol{q}, \boldsymbol{k} j i}^{x *}\right) \\
\left.\cdot \omega_{\boldsymbol{k} i} \omega_{\boldsymbol{k}+\boldsymbol{q} j} \frac{n_{\boldsymbol{k}+\boldsymbol{q} j}-n_{\boldsymbol{k} i}}{\omega_{\boldsymbol{k} i}-\omega_{\boldsymbol{k}+\boldsymbol{q} j}}\right|_{\boldsymbol{q} \rightarrow 0}, \quad(\mathrm{~A}
\end{array}
$$

where $S_{\boldsymbol{k}+\boldsymbol{q}, \boldsymbol{k} j i} \equiv \bar{\psi}_{\boldsymbol{k}+\boldsymbol{q} j} \psi_{\boldsymbol{k} i}$ and $\boldsymbol{V}_{\boldsymbol{k}, \boldsymbol{k}+\boldsymbol{q} i j} \equiv$ $\bar{\psi}_{\boldsymbol{k} i} \frac{\partial\left(\tilde{H}_{\boldsymbol{k}}+\tilde{H}_{\boldsymbol{k}+\boldsymbol{q}}\right)}{\partial \boldsymbol{k}} \psi_{\boldsymbol{k}+\boldsymbol{q} j}+\psi_{\boldsymbol{k} i}^{\dagger} \frac{\partial\left(\tilde{H}_{\boldsymbol{k}}^{\dagger}+\tilde{H}_{\boldsymbol{k}+\boldsymbol{q}}^{\dagger}\right)}{\partial \boldsymbol{k}} \bar{\psi}_{\boldsymbol{k}+\boldsymbol{q} j}^{\dagger}$.

First, we calculate the inter-band contribution from the terms with $i \neq j$ in Eq. (A34). When $\boldsymbol{q} \rightarrow 0$, we have:

$\tilde{M}_{E}^{z, \text { inter }}=-\frac{\hbar}{8} \sum_{\boldsymbol{k} ; i, j=1}^{6 r} \frac{\operatorname{Im}\left(\frac{\partial \bar{\psi}_{\boldsymbol{k} j}}{\partial k_{y}} \psi_{\boldsymbol{k} i} \mathcal{V}_{\boldsymbol{k} i j}^{x}\right) \omega_{\boldsymbol{k} i} \omega_{\boldsymbol{k} j}\left(n_{\boldsymbol{k} j}-n_{\boldsymbol{k} i}\right)}{\omega_{\boldsymbol{k} i}-\omega_{\boldsymbol{k} j}}$

We further have:

$\operatorname{Im}\left(\frac{\partial \bar{\psi}_{\boldsymbol{k} j}}{\partial k_{y}} \psi_{\boldsymbol{k} i} \mathcal{V}_{\boldsymbol{k} i j}^{x}\right)=\frac{1}{2} \operatorname{Im}\left(\frac{\partial \bar{\psi}_{\boldsymbol{k} j}}{\partial k_{y}} \psi_{\boldsymbol{k} i} \mathcal{V}_{\boldsymbol{k} i j}^{x}-\psi_{\boldsymbol{k} i}^{\dagger} \frac{\partial \bar{\psi}_{\boldsymbol{k} j}^{\dagger}}{\partial k_{y}} \mathcal{V}_{\boldsymbol{k} i j}^{x *}\right)$

Noting $\mathcal{V}_{\boldsymbol{k} i j}^{*}=\mathcal{V}_{\boldsymbol{k} j i}$ and inserting Eq. A36 into Eq. (A35), we obtain:

$$
\begin{aligned}
\tilde{M}_{E}^{z, \text { inter }}=-\frac{\hbar}{16} \sum_{\boldsymbol{k} ; i, j=1}^{6 r} \operatorname{Im} & {\left[\left(\frac{\partial \bar{\psi}_{\boldsymbol{k} j}}{\partial k_{y}} \psi_{\boldsymbol{k} i}-\psi_{\boldsymbol{k} j}^{\dagger} \frac{\partial \bar{\psi}_{\boldsymbol{k} i}^{\dagger}}{\partial k_{y}}\right) \mathcal{V}_{\boldsymbol{k} i j}^{x}\right] } \\
& \frac{\omega_{\boldsymbol{k} i} \omega_{\boldsymbol{k} j}\left(n_{\boldsymbol{k} j}-n_{\boldsymbol{k} i}\right)}{\omega_{\boldsymbol{k} i}-\omega_{\boldsymbol{k} j}},
\end{aligned}
$$

where we have interchanged $i$ and $j$ of the second term in Eq. (A36). Using Eq. (A27), we have

$$
\begin{aligned}
& \tilde{M}_{E}^{z, \text { inter }}=\frac{\hbar}{32} \sum_{\boldsymbol{k} ; i, j=1}^{6 r} \frac{\operatorname{Im}\left(\mathcal{V}_{\boldsymbol{k} j i}^{y} \mathcal{V}_{\boldsymbol{k} i j}^{x}\right) \omega_{\boldsymbol{k} i} \omega_{\boldsymbol{k} j}\left(n_{\boldsymbol{k} j}-n_{\boldsymbol{k} i}\right)}{\left(\omega_{\boldsymbol{k} i}-\omega_{\boldsymbol{k} j}\right)^{2}}, \\
& =-\frac{\hbar}{32} \sum_{\boldsymbol{k} ; i, j=1}^{6 r} \frac{\operatorname{Im}\left(\mathcal{V}_{\boldsymbol{k} i j}^{x} \mathcal{V}_{\boldsymbol{k} j i}^{y}-\mathcal{V}_{\boldsymbol{k} i j}^{y} \mathcal{V}_{\boldsymbol{k} j i}^{x}\right) \omega_{\boldsymbol{k} i} \omega_{\boldsymbol{k} j} n_{\boldsymbol{k} i}}{\left(\omega_{\boldsymbol{k} i}-\omega_{\boldsymbol{k} j}\right)^{2}} .
\end{aligned}
$$

Comparing Eq. (A38) and (A25), we finally obtain:

$$
\begin{aligned}
\tilde{M}_{E}^{z, \text { inter }}= & -\frac{\hbar}{2} \operatorname{Im} \sum_{\boldsymbol{k} ; i=1}^{3 r}\left(\frac{\partial \bar{\psi}_{\boldsymbol{k} i}}{\partial k_{x}} \tilde{H}_{\boldsymbol{k}} \frac{\partial \psi_{\boldsymbol{k} i}}{\partial k_{y}}-\frac{\partial \bar{\psi}_{\boldsymbol{k} i}}{\partial k_{y}} \tilde{H}_{\boldsymbol{k}} \frac{\partial \psi_{\boldsymbol{k} i}}{\partial k_{x}}\right) \\
& \cdot \omega_{\boldsymbol{k} i}\left(2 n_{\boldsymbol{k} i}+1\right) \\
= & -\frac{\hbar}{2} \sum_{\boldsymbol{k} ; i=1}^{3 r} \mathcal{M}_{\boldsymbol{k} i}^{z} \omega_{\boldsymbol{k} i}\left(2 n_{\boldsymbol{k} i}+1\right) .
\end{aligned}
$$


Second, we calculate the intra-band contribution from the term with $i=j$ in Eq. (A34):

$$
\begin{aligned}
\tilde{M}_{E}^{z, \text { intra }}=-\frac{\hbar}{16} & \frac{\partial}{\partial q_{y}} \sum_{\boldsymbol{k} ; i=1}^{6 r} \operatorname{Im}\left[S_{\boldsymbol{k}+\boldsymbol{q}, \boldsymbol{k} i}\left(\mathcal{V}_{\boldsymbol{k}, \boldsymbol{k}+\boldsymbol{q} i}^{x}+\mathcal{V}_{\boldsymbol{k}+\boldsymbol{q}, \boldsymbol{k} i}^{x *}\right)\right] \\
& \left.\frac{\omega_{\boldsymbol{k} i} \omega_{\boldsymbol{k}+\boldsymbol{q} i}\left(n_{\boldsymbol{k}+\boldsymbol{q} i}-n_{\boldsymbol{k} i}\right)}{\omega_{\boldsymbol{k} i}-\omega_{\boldsymbol{k}+\boldsymbol{q} i}}\right|_{\boldsymbol{q} \rightarrow 0} . \quad(\mathrm{A} 41)
\end{aligned}
$$

For further simplification, note:

$$
\begin{gathered}
\left.\frac{\partial S_{\boldsymbol{k}+\boldsymbol{q}, \boldsymbol{k} i}}{\partial q_{y}}\right|_{\boldsymbol{q} \rightarrow 0}=\frac{\partial \bar{\psi}_{\boldsymbol{k} i}}{\partial k_{y}} \psi_{\boldsymbol{k} i}, \\
\left.\frac{\partial\left(\mathcal{V}_{\boldsymbol{k}, \boldsymbol{k}+\boldsymbol{q} i}^{x}+\mathcal{V}_{\boldsymbol{k}+\boldsymbol{q}, \boldsymbol{k} i}^{x *}\right)}{\partial q_{y}}\right|_{\boldsymbol{q} \rightarrow 0}=2\left(2 \bar{\psi}_{\boldsymbol{k} i} \frac{\partial \tilde{H}_{\boldsymbol{k}}}{\partial k_{x}} \frac{\partial \psi_{\boldsymbol{k} i}}{\partial k_{y}}\right. \\
+2 \psi_{\boldsymbol{k} i}^{\dagger} \frac{\left.\partial \tilde{H}_{\boldsymbol{k}}^{\dagger} \frac{\partial \bar{\psi}_{\boldsymbol{k} i}^{\dagger}}{\partial k_{x}}+\bar{\psi}_{\boldsymbol{k} i} \frac{\partial^{2} \tilde{H}_{\boldsymbol{k}}}{\partial k_{x} \partial k_{y}} \psi_{\boldsymbol{k} i}+\psi_{\boldsymbol{k} i}^{\dagger} \frac{\partial^{2} \tilde{H}_{\boldsymbol{k}}^{\dagger}}{\partial k_{x} \partial k_{y}} \bar{\psi}_{\boldsymbol{k} i}^{\dagger}\right)}{(\mathrm{A} 42)} \\
\left.\frac{n_{\boldsymbol{k}+\boldsymbol{q} i}-n_{\boldsymbol{k} i}}{\omega_{\boldsymbol{k}+\boldsymbol{q} i}-\omega_{\boldsymbol{k} i}}\right|_{\boldsymbol{q} \rightarrow 0}=n_{\boldsymbol{k} i}^{\prime} .
\end{gathered}
$$

One can show that the sum of the last two terms in Eq. A42 is real, so it has no contribution. So we can write:

$$
\begin{aligned}
\tilde{M}_{E}^{z, \text { intra }}=\frac{\hbar}{4} \sum_{\boldsymbol{k} ; i=1}^{6 r} \operatorname{Im} & {\left[2 \frac{\partial \bar{\psi}_{\boldsymbol{k} i}}{\partial k_{y}} \psi_{\boldsymbol{k} i} \frac{\partial \omega_{\boldsymbol{k} i}}{\partial k_{x}}+\bar{\psi}_{\boldsymbol{k} i} \frac{\partial \tilde{H}_{\boldsymbol{k}}}{\partial k_{x}} \frac{\partial \psi_{\boldsymbol{k} i}}{\partial k_{y}}\right.} \\
& \left.+\psi_{\boldsymbol{k} i}^{\dagger} \frac{\partial \tilde{H}_{\boldsymbol{k}}^{\dagger}}{\partial k_{x}} \frac{\partial \bar{\psi}_{\boldsymbol{k} i}^{\dagger}}{\partial k_{y}}\right] \omega_{\boldsymbol{k} i}^{2} n_{\boldsymbol{k} i}^{\prime}, \quad(\mathrm{A} 43)
\end{aligned}
$$

With $\operatorname{Im}\left[\frac{\partial \bar{\psi}_{\boldsymbol{k} i}}{\partial k_{y}} \psi_{\boldsymbol{k} i}\right]=-\operatorname{Im}\left[\bar{\psi}_{\boldsymbol{k} i} \frac{\partial \psi_{\boldsymbol{k} i}}{\partial k_{y}}\right]=\operatorname{Im}\left[\frac{\partial \psi_{\boldsymbol{k} i}^{\dagger}}{\partial k_{y}} \bar{\psi}_{\boldsymbol{k} i}^{\dagger}\right]$, we come to

$$
\begin{aligned}
\tilde{M}_{E}^{z, \text { intra }}= & \frac{\hbar}{4} \sum_{\boldsymbol{k} ; i=1}^{6 r} \operatorname{Im}\left[\bar{\psi}_{\boldsymbol{k} i} \frac{\partial\left(\tilde{H}_{\boldsymbol{k}}-\omega_{\boldsymbol{k} i}\right)}{\partial k_{x}} \frac{\partial \psi_{\boldsymbol{k} i}}{\partial k_{y}}\right. \\
& \left.+\psi_{\boldsymbol{k} i}^{\dagger} \frac{\partial\left(\tilde{H}_{\boldsymbol{k}}^{\dagger}-\omega_{\boldsymbol{k} i}\right)}{\partial k_{x}} \frac{\partial \bar{\psi}_{\boldsymbol{k} i}^{\dagger}}{\partial k_{y}}\right] \omega_{\boldsymbol{k} i}^{2} n_{\boldsymbol{k} i}^{\prime}
\end{aligned}
$$

Using $\quad \bar{\psi}_{\boldsymbol{k} i} \frac{\partial\left(\tilde{H}_{\boldsymbol{k}}-\omega_{\boldsymbol{k} i}\right)}{\partial k_{x}}=-\frac{\partial \bar{\psi}_{\boldsymbol{k} i}}{\partial k_{x}}\left(\tilde{H}_{\boldsymbol{k}}-\omega_{\boldsymbol{k} i}\right)$ and $\psi_{\boldsymbol{k} i}^{\dagger} \frac{\partial\left(\tilde{H}_{\boldsymbol{k}}^{\dagger}-\omega_{\boldsymbol{k} i}\right)}{\partial k_{x}}=-\frac{\partial \psi_{\boldsymbol{k} i}^{\dagger}}{\partial k_{x}}\left(\tilde{H}_{\boldsymbol{k}}^{\dagger}-\omega_{\boldsymbol{k} i}\right)$, we obtain:

$$
\begin{aligned}
\tilde{M}_{E}^{z, \text { intra }} & =-\frac{\hbar}{4} \sum_{\boldsymbol{k} ; i=1}^{6 r} \operatorname{Im}\left[\frac{\partial \bar{\psi}_{\boldsymbol{k} i}}{\partial k_{x}}\left(\tilde{H}_{\boldsymbol{k}}-\omega_{\boldsymbol{k} i}\right) \frac{\partial \psi_{\boldsymbol{k} i}}{\partial k_{y}}\right. \\
& \left.-\frac{\partial \bar{\psi}_{\boldsymbol{k} i}}{\partial k_{y}}\left(\tilde{H}_{\boldsymbol{k}}-\omega_{\boldsymbol{k} i}\right) \frac{\partial \psi_{\boldsymbol{k} i}}{\partial k_{x}}\right] \omega_{\boldsymbol{k} i}^{2} n_{\boldsymbol{k} i}^{\prime} \cdot
\end{aligned}
$$

Using the definitions for $\mathcal{M}_{\boldsymbol{k} i}^{z}$ and $\Omega_{\boldsymbol{k} i}^{z}$, we have:

$$
\begin{aligned}
\tilde{M}_{E}^{z, \text { intra }} & =-\frac{\hbar}{4} \sum_{\boldsymbol{k} ; i=1}^{6 r}\left(\mathcal{M}_{\boldsymbol{k} i}^{z}+\omega_{\boldsymbol{k} i} \Omega_{\boldsymbol{k} i}^{z}\right) \omega_{\boldsymbol{k} i}^{2} n_{\boldsymbol{k} i}^{\prime} \\
& =-\frac{\hbar}{2} \sum_{\boldsymbol{k} ; i=1}^{3 r}\left(\mathcal{M}_{\boldsymbol{k} i}^{z}+\omega_{\boldsymbol{k} i} \Omega_{\boldsymbol{k} i}^{z}\right) \omega_{\boldsymbol{k} i}^{2} n_{\boldsymbol{k} i}^{\prime}
\end{aligned}
$$

Finally, combining Eqs. A40 and (A47), we come to Eq. (19).
1 C. Strohm, G.L.J.A. Rikken, and P. Wyder, Phys. Rev. Lett. 95, 155901 (2005).

2 A. V. Inyushkin and A. N. Taldenkov, JETP Lett. 86, 379 (2007).

${ }^{3}$ L. Sheng, D. N. Sheng, and C. S. Ting, Phys. Rev. Lett. 96, 155901 (2006).

4 Y. Kagan and L. A. Maksimov, Phys. Rev. Lett. 100, 145902 (2008).

5 J.-S. Wang and L. Zhang, Phys. Rev. B 80, 012301 (2009).

${ }^{6}$ L. Zhang, J. Ren, J.-S. Wang and B. Li, Phys. Rev. Lett. 105, 225901 (2010).

7 B. K. Agarwalla, L. Zhang, J.-S. Wang and B. Li, Eur. Phys. J. B 81, 197 (2011).

8 R. de. L. Kronig, Physica, 5, 33(1939).

9 T. Jungwirth, Q. Niu, and A. H. MacDonald, Phys. Rev.
Lett. 88, 207208 (2002).

10 L. Zhang, J. Ren, J.-S. Wang and B. Li, J. Phys.: Condens. Matter 23, 305402 (2011).

11 E. Prodan and C. Prodan, Phys. Rev. Lett. 103, 248101 (2009).

12 C. A. Mead and D. G. Truhlar, J. Chem. Phys. 70, 2284 (1979).

13 T. Qin, Q. Niu and J. R. Shi, Phys. Rev. Lett. 107, 236601 (2011).

14 N. R. Cooper, B. I. Halperin and I. M. Ruzin, Phys. Rev. B 55, 2344 (1997).

15 R. Jackiw, Int. J. Mod. Phys. A3, 285 (1988).

16 R. Resta, J. Phys.: Condens. Matter 12, R107(2000).

17 A. Holz, IL Nuovo Cimento 9B, 83(1972).

18 J. Callaway, Quantum Theory of the Solid State, 2nd ed. 
(Academic Press, San Diego, 1991).

19 G. Sundaram and Q. Niu, Phys. Rev. B 59, 14915 (1999).

20 J. M. Luttinger, Phys. Rev. 135, A1505 (1964).

21 R. Kubo, M. Toda and N. Hashitsume, Statistical Physics II (Springer-Verlag, Berlin, 1983).

22 R. J. Hardy, Phys. Rev. 132, 168 (1963).

23 B. I. Halperin, Jpn. J. Appl. Phys. 26S3, 1913 (1987).

24 L.G.C. Rego and G. Kirczenow, Phys. Rev. Lett. 81, 232(1998); M.P. Blencowe, Phys. Rev. B 59, 4992(1999).

${ }^{25}$ K. Schwab, E.A. Henriksen, J.M. Worlock and M.L. Roukes, Nature 404, 974(2000).

26 T. Yamamoto, K. Watanabe and K. Mii, Phys. Rev. B 70,
245402(2004).

27 L.D. Landau and E.M. Lifshitz, Theory of Elasticity, 3rd ed. (Butterworth-Heinemann, Amsterdam, 1986).

28 A.A. Kovalev, J. Sinova, and Y. Tserkovnyak, Phys. Rev. Lett. 105, 036601(2010)

29 N. Nagaosa, J. Sinova, S. Onoda, A.H. MacDonald and N.P. Ong, Rev. Mod. Phys. 82, 1539(2010).

30 A. V. Inyushkin and A. N. Taldenkov, JETP Lett. 111, 760 (2010).

31 J.K. Flicker and P.L. Leath, Phys. Rev. B 7, 2296(1973). 